\title{
Tumor-suppressive function of EZH2 is through inhibiting glutaminase
}

\author{
Yongfeng Liu ${ }^{1,4}{ }^{凶}$, Cheng-e $\mathrm{Tu}^{1,4}$, Xuxue Guo ${ }^{1,4}$, Changjie Wu ${ }^{1}$, Chuncai Gu ${ }^{1}$, Qiuhua Lai ${ }^{1}$, Yuxin Fang ${ }^{1}$, Junqi Huang ${ }^{2}$, \\ Zhizhang Wang $\left(\mathbb{D}^{3 \times}\right.$, Aimin Li $\mathbb{B D}^{1 \times}$ and Side Liu (iD)
}

(c) The Author(s) 2021

Tumors can use metabolic reprogramming to survive nutrient stress. Epigenetic regulators play a critical role in metabolic adaptation. Here we screened a sgRNA library to identify epigenetic regulators responsible for the vulnerability of colorectal cancer (CRC) cells to glucose deprivation and found that more EZH2-knockout cells survived glucose deprivation. Then, we showed that $\mathrm{EZH} 2$ expression was significantly downregulated in response to glucose deprivation in a glucose-sensitive CRC cell line, and EZH2knockdown cells were more resistant to glucose deprivation. Mechanistically, EZH2 deficiency upregulated the expression of glutaminase (GLS) and promoted the production of glutamate, which in turn led to increased synthesis of intracellular glutathione (GSH) and eventually attenuated the reactive oxygen species (ROS)-mediated cell death induced by glucose deprivation. Although $\mathrm{EZH} 2$ functioned as an oncogene in cancer progression and $\mathrm{EZH} 2$ knockout abolished colorectal cancer development in a mouse model, here we revealed a mechanistic link between EZH2 and metabolic reprogramming via the direct regulation of GLS expression and observed a negative correlation between EZH2 and GLS expression in colorectal cancer tissues. These findings further confirmed the importance of heterogeneity, provided an explanation for the clinical tolerance of cancer cells to EZH2 inhibitors from the perspective of metabolism, and proposed the possibility of combining EZH2 inhibitors and glutamine metabolism inhibitors for the treatment of cancer.

Cell Death and Disease (2021)12:975; https://doi.org/10.1038/s41419-021-04212-7

\section{INTRODUCTION}

Studies of metabolic vulnerability have identified many therapeutic targets for the treatment of cancer [1, 2]. During cancer progression, highly proliferative cancer cells are frequently exposed to nutrient-scarce conditions that result from poor vascularization or antiangiogenic therapy; thus, cancer cells need to adapt to nutrient stress, including the stress caused by glucose limitation. Cancer cells transformed by oncogenes, such as MYC, often display glucose addiction, which makes them highly dependent on glucose metabolism for growth and redox homeostasis [3-5], and the exhaustion of glucose in the microenvironment disrupts the intracellular homeostasis of cancer cells, ultimately leading to cell death. The regulation of glucose metabolism has been thoroughly studied in previous research, and more factors that promote glucose addiction have been identified, but factors responsible for adaptation to glucose limitation are relatively rare.

Covalent modifications of histones, including methylation and acetylation, play fundamental roles in the epigenetic regulation of gene expression. Histone methylation can either activate or repress gene transcription, depending on the position of the lysine residue within the histone and the combination of different modifications. Recent genomic studies have enhanced the understanding of the role of histone modifiers in cancer progression [6, 7]. However, the particular epigenetic regulators involved in the adaptation to glucose limitation have rarely been studied.

To study the epigenetic regulation of cancer cells under glucose deprivation conditions, we screened a knockout library of histone modifiers in the glucose-sensitive CRC cell line HCT116. First, the infected HCT116 cells were cultured in a glucose-depleted medium as indicated, and then, the dead and live cells were separated for gRNA sequencing. The results showed that gRNAs that target EZH2 were enriched in the surviving cells, which suggested that $\mathrm{EZH} 2$ might be involved in the cancer cell response to glucose deprivation. EZH2 is the enzymatic subunit of polycomb repressive complex 2 (PRC2), which tri-methylates H3K27 and facilitates chromatin compaction and gene silencing [8]. As an oncogene in solid cancers, EZH2 has been reported to affect many cancer-related signaling pathways and eventually promote cancer cell proliferation, malignant transformation, and survival [9-12].

Mechanistically, we identified glutaminase (GLS) as the direct target gene of EZH2 and showed that its expression could be silenced by $\mathrm{EZH} 2$ under glucose deprivation conditions. When the function of EZH2 is blocked with shRNA or inhibitors, the

\footnotetext{
${ }^{1}$ Guangdong Provincial Key Laboratory of Gastroenterology, Department of Gastroenterology, Nanfang Hospital, Southern Medical University, Guangzhou 510515, China.

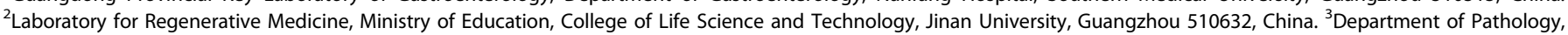

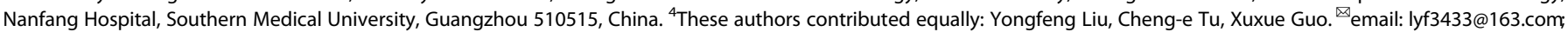
wangzz89@smu.edu.cn; lam0725@163.com; sideliu@smu.edu.cn

Edited by Professor Massimiliano Agostini
}

Received: 13 May 2021 Revised: 26 July 2021 Accepted: 9 September 2021

Published online: 20 October 2021 
repression of GLS transcription is alleviated, and high GLS expression accelerates glutamine metabolism and GSH accumulation, which in turn attenuates glucose-deprivation-induced reactive oxygen species (ROS) production and cell death. In summary, these data suggest a tumor-suppressive role of EZH2 under glucose deprivation conditions.

\section{RESULTS \\ Conditional screening reveals that EZH2 is involved in the adaptation to glucose starvation}

Increasing evidence shows that metabolic reprogramming is an important adaptive mechanism that facilitates the survival and proliferation of cancer cells in nutrient-deficient tumor microenvironments [13], and epigenetic regulators play a critical role in this mechanism [14]. First, we investigated the sensitivity of different CRC cell lines to glucose starvation. The results showed that glucose starvation induced significant cell death, as indicated by the levels of cleaved PARP (C-PARP), only in glucose-sensitive cell lines (such as HCT116 and SW480 cells) but not in RKO and LoVo cells (Fig. 1A). Therefore, to identify the epigenetic factors responsible for adaptation to metabolic stress during glucose deprivation, we conducted genetic screening in HCT116 cells by using a sgRNA library targeting histone modifiers, including "writers", "erasers", and "readers" of acetylation and methylation (Fig. 1B, Supplementary Table 1). HCT116 cells were infected with the library, and cultured without glucose for $16 \mathrm{~h}$. The suspended dead cells were harvested, and the rest cells were digested by trypsin and re-cultured in DMEM supplemented with $2.5 \mathrm{mM}$ glucose for another $10 \mathrm{~h}$ to further separate dead cells attached to live cells. Then the cells were separated into two groups: the unattached dead cells (group $S$, sensitive to glucose deprivation) and the attached live cells (group $\mathrm{R}$, resistance to glucose deprivation). The separated cells were subjected to genomic DNA isolation and deep sequencing of the integrated sgRNAs. After analysis, 262 candidate gene sgRNAs were enriched in group $\mathrm{R}$ compared with group $\mathrm{S}$ (fold change $>1.5$ ) (Supplementary Table 2). By comparing these candidate genes with the downregulated genes in HCT116 cells exposed to glucose deprivation (2798 genes), we obtained a list of 41 genes, among which EZH2 stood out (Fig. 1C and Supplementary Table 3). Further analysis showed that sgRNAs that target other subunits of polycomb repressive complex 2 (PRC2), such as EED, SUZ12, and PHF19, also tended to be enriched in group R (Supplementary Fig. $1 \mathrm{~A})$. These results led us to wonder whether the expression of $\mathrm{EZH} 2$ in HCT116 cells could be downregulated to adapt to glucose deprivation. To test this idea, CRC cells were cultured with or without glucose, and the expression of EZH2, SUZ12, and EED was detected. The results showed that glucose deprivation decreased EZH2 mRNA levels in a dose- and time-dependent manner in the glucose-sensitive CRC cell lines HCT116 and SW480 (Fig. 1D, E and Supplementary Fig. 1B, C) but not in the glucose-insensitive cell lines RKO and LoVo (Supplementary Fig. 1D, E). Additionally, similar results were obtained when the $\mathrm{EZH} 2$ protein levels were examined (Fig. 1F and Supplementary Fig. 2B). These data suggest that downregulation of EZH2 expression in glucose-sensitive CRC cells may be a strategy by which some cancer cells adapt to glucose deprivation.

\section{Downregulation of EZH2 expression alleviates glucose dependence in glucose-sensitive CRC cells}

After our previous work on the function of EZH2 in colitis [15], we conducted related studies on the function of $\mathrm{EZH} 2$ in colorectal cancer. Using the APC ${ }^{\mathrm{in} /+}$ and AOM/DSS models, we showed that the deletion of the EZH2 gene significantly inhibited the occurrence of colon cancer, indicating a cancer-promoting function of $\mathrm{EZH} 2$ in $\mathrm{CRC}$ (Supplementary Fig. $3 \mathrm{~A}-\mathrm{H}$ ); these results were consistent with the function of EZH2 in other solid cancers $[11,16,17]$. However, the paradoxical results in the screening described above suggested that EZH2 might perform different functions under stressful conditions, such as glucose deprivation, and made us wonder whether EZH2 could play an unconventional role in metabolism. To test this possibility, we generated EZH2-knockdown CRC cells and cultured them $a$ in glucose-free medium. Observations of cell morphology revealed that the cell death in HCT116 cells and the cell cycle arrest in RKO cells caused by glucose deprivation were both attenuated by EZH2 knockdown (Fig. 2A-C). Next, we further confirmed the cell death phenotype of HCT116 cells by analyzing the expression of C-PARP, which is a key indicator of apoptosis. The increased levels of C-PARP observed under glucose deprivation conditions were attenuated by the knockdown of EZH2 (Fig. 2D). Similar experiments in SW480 cells also yielded the same results (Fig. $2 \mathrm{E})$. To test the function of EZH2 enzymatic activity in glucosedeprivation-induced cell death, the EZH2 inhibitor GSK126 was used to interfere with its enzyme activity. The results showed that the cell apoptosis induced by glucose deprivation was significantly ameliorated in HCT116 and SW480 cells pretreated with GSK126 for $48 \mathrm{~h}$ (Fig. 2F, G). Correspondingly, the overexpression of $E Z H 2$ in CRC cells significantly enhanced the sensitivity of CRC cells to glucose deprivation, i.e., EZH2 overexpression promoted the cell death of glucose-sensitive cells and the cell cycle arrest of glucose-insensitive cells (Fig. $2 \mathrm{H}$ and Supplementary Fig. 2A). Consistently, EZH2 deficiency in HCT116 and SW480 cells attenuated the cell death caused by glucose deprivation, resulting in more live cells (Fig. 2l-K). Together, our data show that EZH2 deficiency alleviates the glucose dependence of CRC cells.

\section{EZH2 deletion promotes GLS expression and inhibits the glucose-deprivation-induced downregulation of GLS expression in glucose-sensitive CRC cells}

Next, we tried to analyze the relevant mechanisms by which EZH2 affects the glucose vulnerability of CRC cells. First, we investigated whether EZH2, a transcriptional regulator, could affect the expression of genes related to glucose metabolism. The RT-qPCR results and the subsequent RNA-Seq results showed that EZH2 deficiency could not regulate the expression of key enzymes of glucose metabolism (Supplementary Fig. 4A-C). The use of the glucose tracer 2-NBDG [18] also revealed that the knockdown of EZH2 did not affect glucose uptake by CRC cells (Supplementary Fig. 4D-G). To determine the molecular mechanism by which EZH2 regulates glucose vulnerability, we performed RNA-Seq analysis with two pairs of cell samples (HCT116 ${ }^{\mathrm{NC}}$ vs. HCT1 $16^{\text {shEZH2 }}$ and $\mathrm{RKO}^{\mathrm{NC}}$ vs. RKO ${ }^{\text {shEZH2 }}$ ). Intersection analysis of the upregulated genes (fold change $>1.2$ ) in the RNA-Seq data combined with the SW480 sequencing results in the GSE118593 dataset revealed that the expression of 224 genes was upregulated in the three cell lines due to EZH2 deletion (Supplementary Fig. 5A) [19]. KEGG pathway analysis of these 224 genes showed that FoxO signaling, Hippo signaling, TGF- $\beta$ signaling, and other signaling pathways were significantly enriched (Fig. 3A). The first pathway that caught our attention was the Hippo signaling pathway, which negatively regulates the transcriptional coactivator Yap to affect cell proliferation and cell survival [20]. We hypothesized that the Hippo-Yap pathway might perform a function in glucose-deprivation-induced cell death similar to its function in ferroptosis; i.e., Yap activity is positively correlated with the cell death phenotype [21]. However, knocking down Yap expression significantly promoted the cell apoptosis induced by glucose deprivation (Supplementary Fig. $5 B-D)$, and this finding could not explain our phenotype. A pathway named "glutamatergic synapse" then attracted our interest; in this pathway, the expression of glutaminase (GLS) appeared to be significantly increased (Fig. 3A, B). GLS is a key enzyme of glutamine metabolism that catalyzes the conversion of glutamine to glutamate [22]. Notably, the mRNA and protein levels of GLS were 
A

B
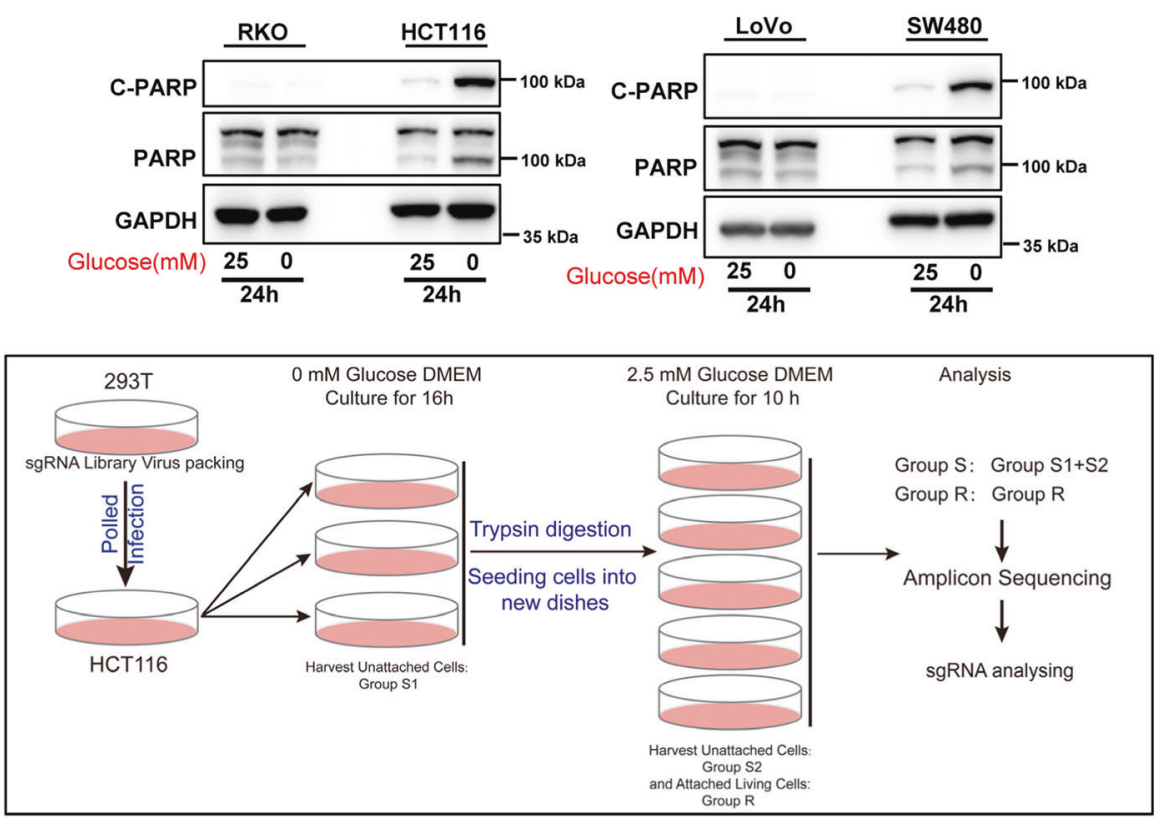

C

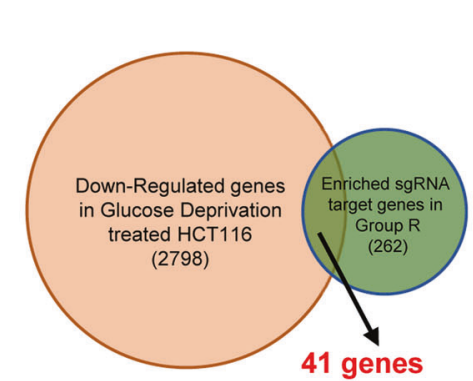

D

HCT116

SW480
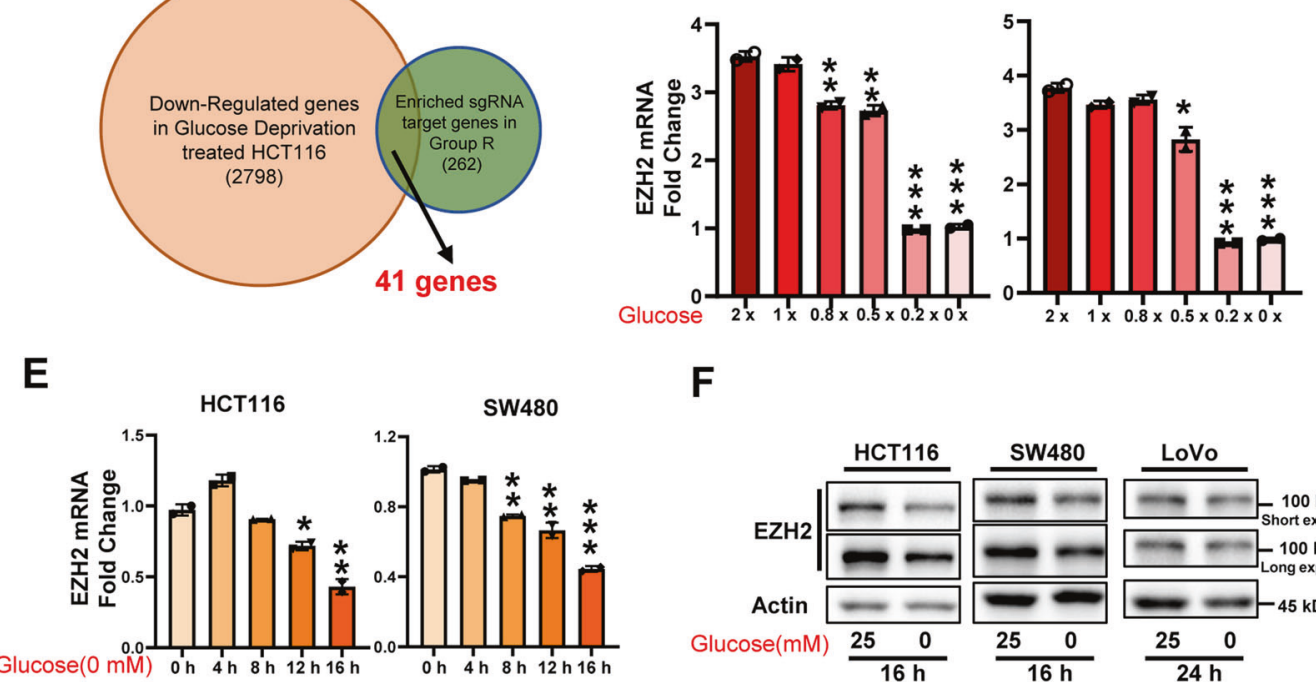

F

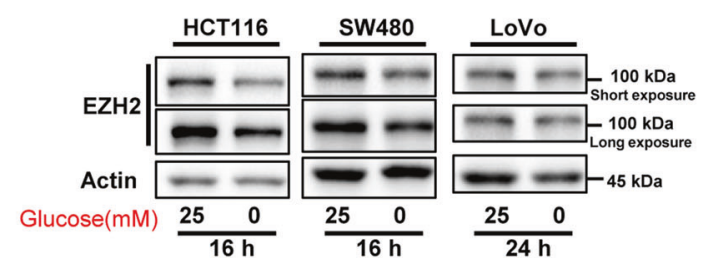

Fig. 1 Library screening shows that EZH2 expression is regulated by glucose supply. A Immunoblotting for cleaved-PARP and PARP expression in indicated colorectal cancer (CRC) cells under glucose deprivation. GAPDH is used as a loading control. B Schematic diagram of library screening under glucose deprivation. C Venn diagram shows the overlap between genes downregulated by glucose deprivation treatment $(n=2798)$, and genes targeted by sgRNAs that enriched in Group R $(n=262)$. D, E RT-qPCR of EZH2 levels in HCT116 and SW480 cells treated with indicated doses of glucose for $24 \mathrm{~h}$ (D) or under glucose deprivation for the indicated time (E). $1 \times$ glucose, $25 \mathrm{mM}$. F Immunoblotting for $\mathrm{EZH} 2$ expression in indicated $\mathrm{CRC}$ cells under glucose deprivation. Actin is used as a loading control. The experiments in A, F were repeated twice independently with similar results. In $\mathbf{D}, \mathbf{E}$, data are mean \pm s.d., $n=3$ independent experiments; $P$-values were calculated using two-tailed unpaired Student's $t$-test. ${ }^{*} P<0.05,{ }^{* *} P<0.01$, ${ }^{* *} P<0.001$.

increased in EZH2-deficient and GSK126-treated cells cultured under normal conditions (Fig. 3C-E and Supplementary Fig. 2C). When the cells were cultured under glucose deprivation conditions, GLS expression was downregulated in glucose-sensitive CRC cells, and this effect was ameliorated by EZH2 inhibition (Fig. 3F-K and Supplementary Fig. 2D). However, although the mRNA level of GLS was significantly upregulated in RKO cells after EZH2 knockdown, the protein level of GLS remained unchanged, even when we prolonged the time of exposure to glucose deprivation conditions (Fig. 3C and Supplementary Fig. 2B). These phenotypes might be explained by the different requirements for glucose metabolism in glucose-sensitive and glucose-insensitive cells. In glucose-sensitive cells, glucose metabolism might play an essential role in redox homeostasis; therefore, glucose deprivation may ultimately result in ROS accumulation and cell death. However, in glucose-insensitive cells, glucose metabolism is needed to provide energy and intermediate metabolite supplies to maintain cancer cell proliferation. Collectively, our data suggest that $\mathrm{EZH} 2$ is a member of the network of cancer metabolism and that EZH2 deficiency upregulates GLS expression and blocks the glucose-deprivation-induced inhibition of GLS transcription in glucose-sensitive cells. 
A

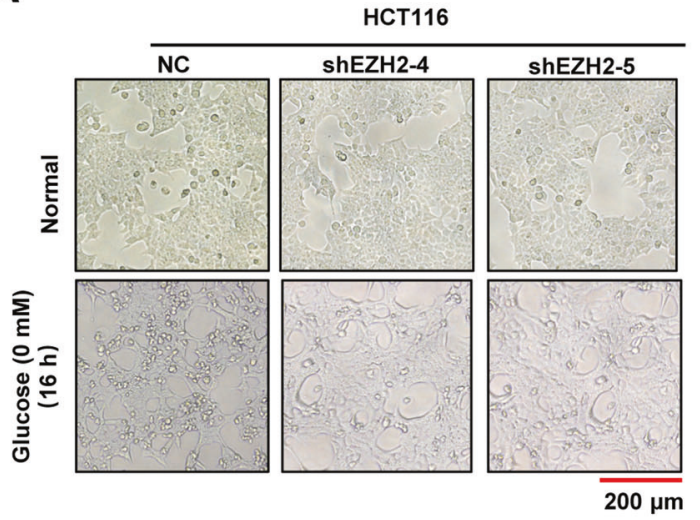

B

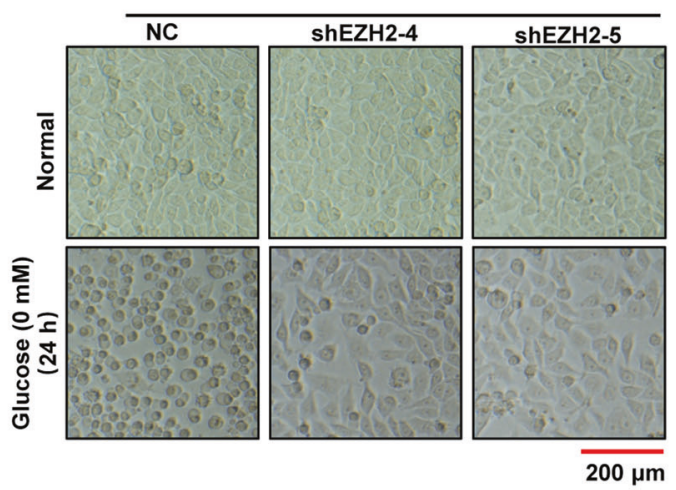

C

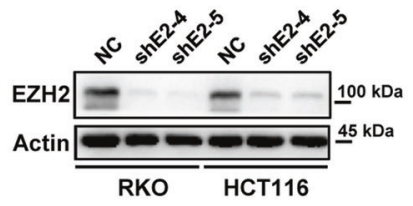

F
D

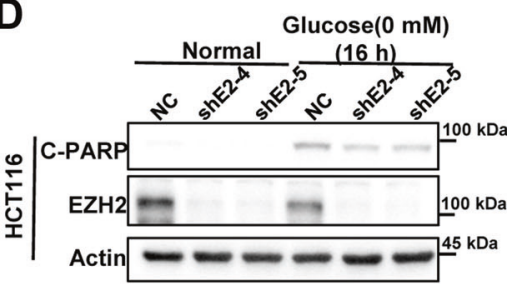

E

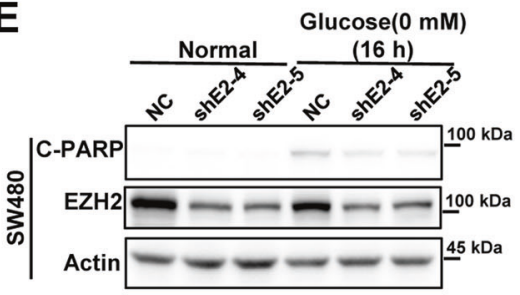

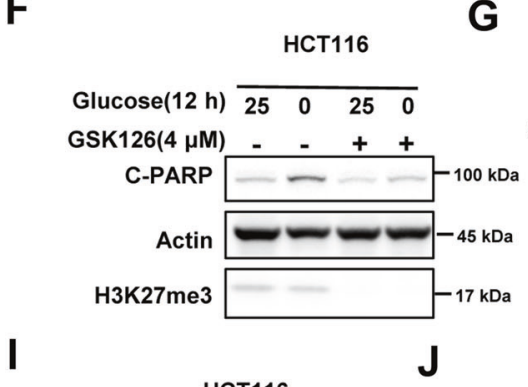

I

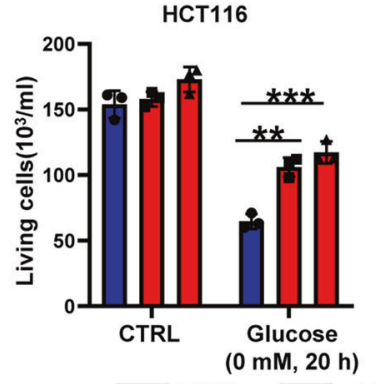

CTRL

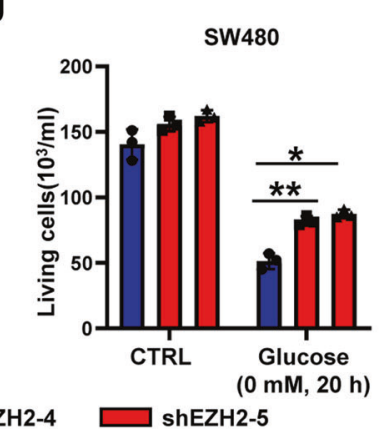

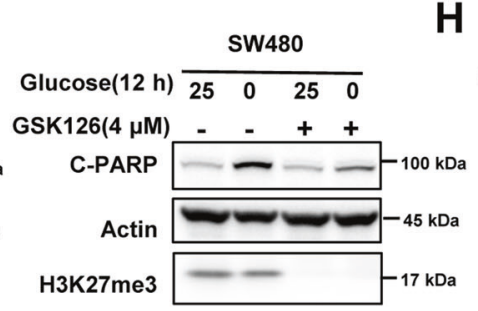

H

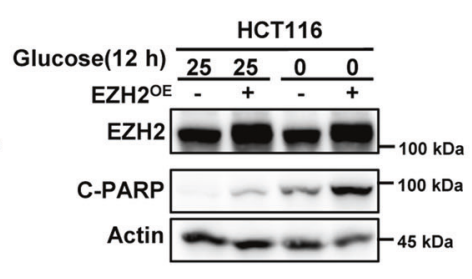

Fig. 2 Absence of EZH2 in glucose-sensitive CRCs enhanced the tolerance to glucose-deprivation-induced cell death. A, B Representative images of HCT116 (A) or RKO (B) NC and shEZH2 cells under normal condition or glucose deprivation. The experiments were repeated twice independently with similar results. C Immunoblotting for EZH2 knockdown efficiency in RKO and HCT116 cells. Actin is used as a loading control. D, E Immunoblotting for cleaved-PARP and EZH2 expression in HCT116 (D) or SW480 (E) NC and shEZH2 cells under normal condition or glucose deprivation $(0 \mathrm{mM}, 16 \mathrm{~h})$. F, G Immunoblotting for cleaved-PARP and H3K27me3 expression in HCT116 (F) or SW480 (G) cells pretreated with or without GSK126 $(4 \mu \mathrm{M}, 48 \mathrm{~h})$, followed by glucose deprivation treatment $(0 \mathrm{mM}, 12 \mathrm{~h})$. $\mathbf{H}$ Immunoblotting for cleaved-PARP and EZH2 expression in HCT116 cells with or without EZH2 overexpression under normal condition or glucose deprivation. I, J Living cell counting of HCT116 (I) or SW480 (J) NC and shEZH2 cells under normal condition or glucose deprivation ( 0 mM, 20 h). K Living cell counting of HCT116 cells pretreated with or without GSK126 $(4 \mu \mathrm{M}, 72 \mathrm{~h})$, cultured under normal condition or glucose deprivation $(0 \mathrm{mM}, 20 \mathrm{~h})$. The experiments in $\mathbf{C}-\mathbf{H}$ were repeated twice independently with similar results. In I, J, K, data are mean \pm s.d., $n=3$ independent experiments; $P$-values were calculated using two-tailed unpaired Student's $t$-test. ${ }^{*} P<0.05,{ }^{* *} P<0.01,{ }^{* * *} P<0.001$. Scale bars, $200 \mu \mathrm{m}$.

GLS is the direct target gene of EZH2 under glucose deprivation conditions and its expression negatively correlates with the expression of EZH2 in CRC tissues

The data described above prompted us to further examine the clinical relevance of the EZH2-GLS relationship in human CRC. We examined the correlation between the expression of $E Z H 2$ and GLS in the GSE24514, GSE14333, and GSE106582 datasets [23, 24].
The results showed that the $E Z H 2$ levels were negatively correlated with those of GLS (Fig. $4 \mathrm{~A}-\mathrm{C}$ ), indicating that GLS could be negatively regulated by $E Z H 2$ during $C R C$ progression. ChIP interrogation of the GLS gene in ENCODE revealed the potential for the recruitment of EZH2 in human neural progenitor cells and hepatocytes (Fig. 4D) and more significant recruitment in mouse intestinal epithelial cells (Fig. 4E) [15, 25]. However, in HCT116 
A

B

C

GIs
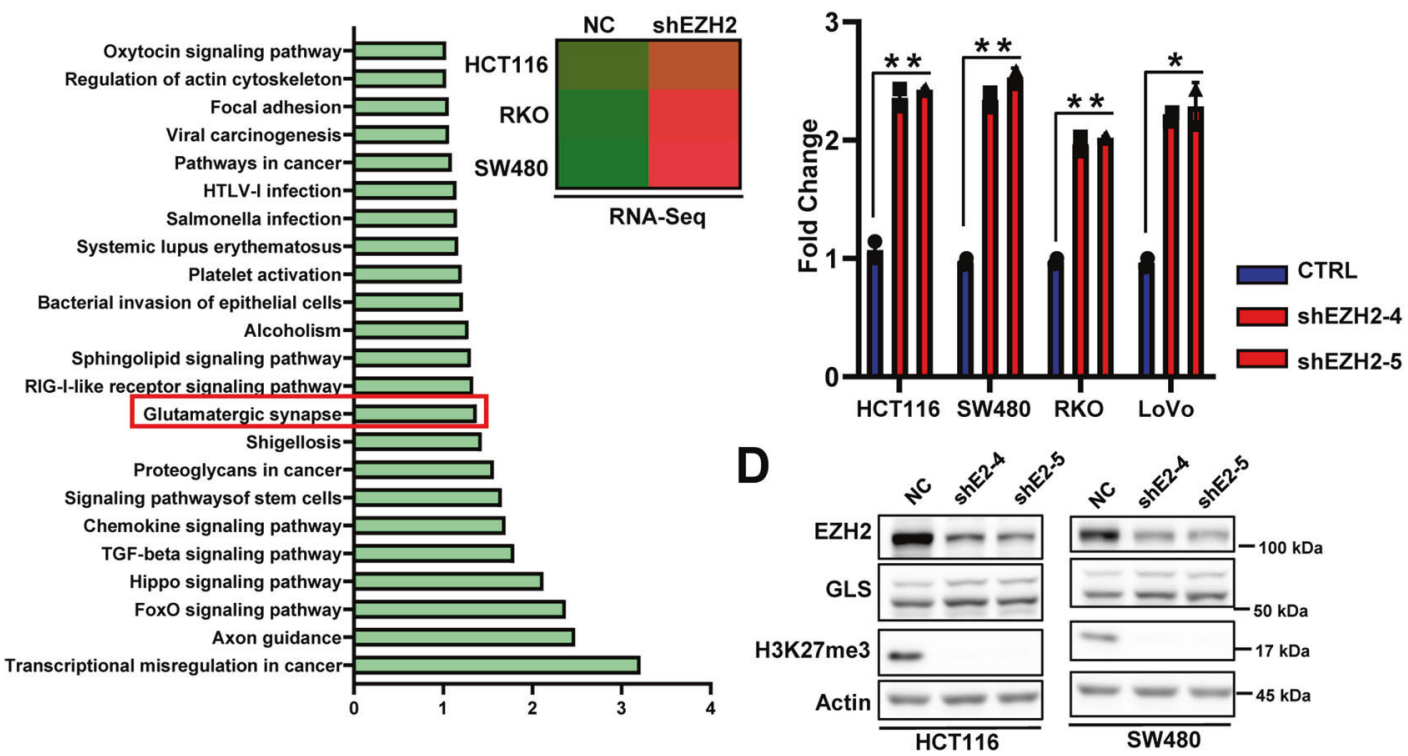

$\mathbf{E}$

E

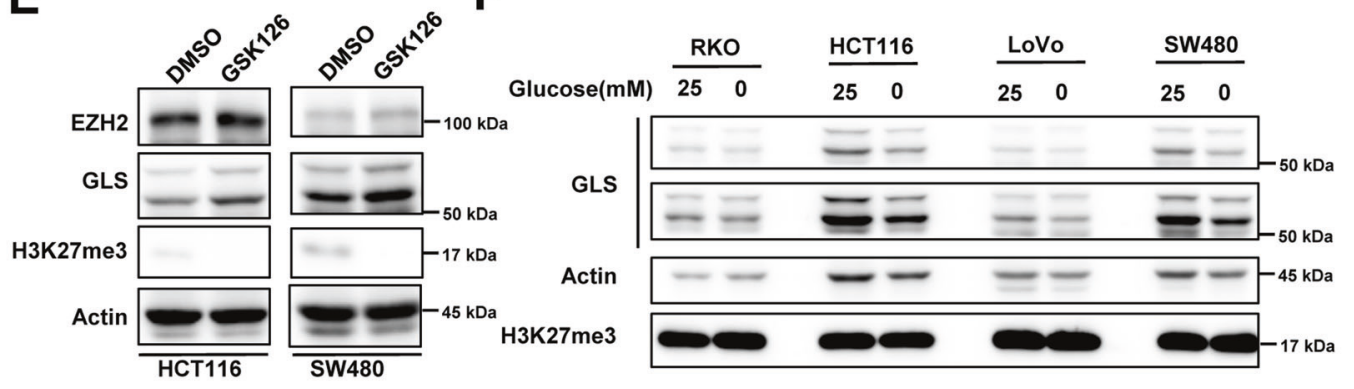

G

H

CTRL $\square$ Glucose (0 mM)(16 h)

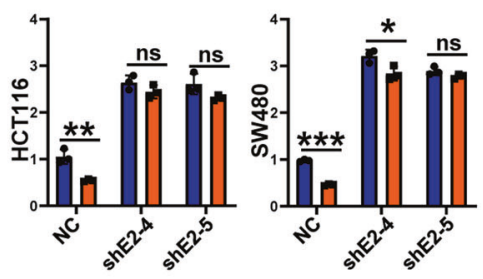

|

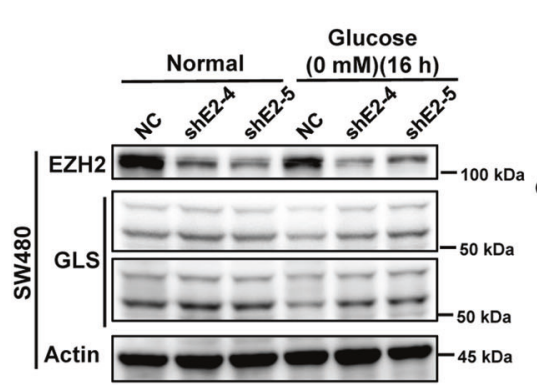

J
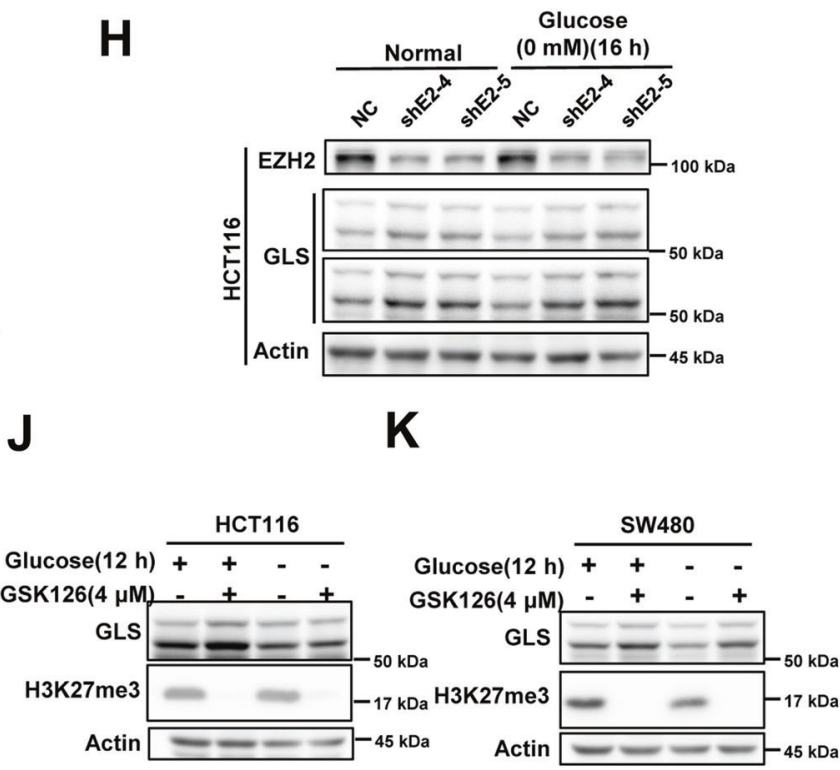

cells, the ENCODE results showed that H3K27Ac exhibited significant peaks in the GLS promoter, while either H3K27me3 or $\mathrm{EZH} 2$ did not in normal culture conditions (Fig. 4F, G and Supplementary Fig. 2E) [25]. This result made us think carefully and put forward the following hypothesis: Under normal conditions, GLS is continuously expressed, as shown by high $\mathrm{H} 3 \mathrm{~K} 27 \mathrm{Ac}$ levels in its promoter. Under glucose deprivation conditions, H3K27Ac is eliminated, and EZH2 gains access to the GLS promoter, increasing the methylation of $\mathrm{H} 2 \mathrm{~K} 27$ and thereby inhibiting the transcription of GLS. Since the GLS promoter had the 
Fig. 3 Absence of EZH2 function attenuates glucose-deprivation-induced downregulation of GLS. A KEGG pathway enrichment analysis showing pathways upregulated by EZH2 deficiency (https://david.ncifcrf.gov/). B RNA-Seq analysis of GLS expression in indicated CRC NC and shEZH2 cells. C RT-qPCR of GLS mRNA levels in indicated CRC NC and shEZH2 cells. D, E Immunoblotting for GLS expression in HCT116 or SW480 cells with (shE2-4/5) or without (NC) EZH2 knockdown (D), or treated with GSK126 (5 $\mu \mathrm{M}, 48 \mathrm{~h})$ (E). Actin is used as a loading control. F Immunoblotting for GLS expression in indicated CRCs under glucose deprivation. G RT-qPCR of GLS mRNA levels in HCT116 or SW480 NC and shEZH2 cells with or without glucose deprivation treatment $(0 \mathrm{mM}, 16 \mathrm{~h})$. $\mathbf{H}, \mathbf{I}$ Immunoblotting for EZH2 and GLS expression in HCT116 (H) or SW480 (I) NC and shEZH2 cells with or without glucose deprivation treatment $(0 \mathrm{mM}, 16 \mathrm{~h})$. J, K Immunoblotting for GLS and H3K27me3 expression in HCT116 (J) or SW480 (K) cells pretreated with or without GSK126 $(4 \mu \mathrm{M}, 48 \mathrm{~h})$, followed by glucose deprivation treatment $(0 \mathrm{mM}, 12 \mathrm{~h})$. The experiments in $\mathbf{D}-\mathbf{F}$ and $\mathbf{H}-\mathbf{K}$ were repeated twice independently with similar results. In $\mathbf{C}, \mathbf{G}$, data are mean \pm s.d., $n=3$ independent experiments; $P$-values were calculated using two-tailed unpaired Student's $t$-test. ${ }^{*} P<0.05$, ${ }^{*} P<0.01$; ns, non-significant.

potential to be directly bound by $\mathrm{EZH} 2$ and the decrease in GLS expression by glucose deprivation was attenuated by EZH2 silencing (Fig. 4D-G and Fig. 3), we sought to investigate whether EZH2 was more inclined to bind to the GLS promoter under glucose deprivation conditions. To test this hypothesis, we first used western blotting to check H3K27Ac and found it could be significantly downregulated under glucose deprivation treatment (Fig. 4H). Then we performed ChIP analysis in HCT116 cells cultured under normal conditions or glucose deprivation conditions and found that $\mathrm{EZH} 2$ could directly bind to the GLS promoter and that the recruitment of EZH2 to the GLS promoter was significantly increased under glucose deprivation conditions (Fig. 4K). As H3K27Ac and H3K27me are antagonistic, we also proved that the modification of H3K27AC in the GLS promoter is decreased (Fig. 4L). Further, we pretreated HCT116 cells with the HDAC inhibitor trichostatin A (TSA). The results showed that the downregulation of GLS expression and the reduction in $\mathrm{H} 3 \mathrm{~K} 27 \mathrm{AC}$ modification in the GLS promoter induced by glucose deprivation were inhibited by TSA treatment (Fig. $4 \mathrm{H}, \mathrm{I}$ ).

Solid tumors exhibit significant heterogeneity in terms of vasculature perfusion. Cells located in the outside layer of the tumor tissue are highly perfused, while the innermost cells are poorly perfused, residing in nutrient-poor environments. Therefore, to further confirm the involvement of the EZH2-GLS axis in the adaptation to glucose deprivation, we performed immunofluorescence staining for EZH2 and GLS in larger colon cancer tissues. The results showed that $\mathrm{EZH} 2$ was highly expressed in the outer layers of tumor tissues, while GLS was highly expressed in the inner layers (Fig. 5A, B). These data indicated that the expression levels of EZH2 and GLS changed dramatically due to different locations in tumor tissues, which explained why the RNA sequencing data did not show a good correlation between EZH2 and GLS expression in CRC (Fig. 4A-C). Taken together, these results suggest that $\mathrm{EZH} 2$ can directly regulate the expression of GLS in CRC and that this correlation is related to the nutritional conditions in the microenvironment.

\section{GLS is involved in the resistance of EZH2-deficient cells to glucose deprivation}

The results described above showed that EZH2 knockdown enhanced the resistance of CRC cells to glucose deprivation and that GLS expression was elevated in EZH2-knockdown cells. Thus, we next investigated whether the resistance of EZH2-deficient cells to glucose deprivation was due to the upregulation of GLS expression. As a key enzyme in glutamine metabolism, GLS has always been an attractive anticancer therapeutic target. A variety of inhibitors that target GLS have entered the clinical trial stage $[26,27]$. Here, bis-2-(5-phenylacetamido-1,2,4-thiadiazol-2-yl) ethyl sulfide (BPTES) was chosen to inhibit the activity of GLS and determine whether the resistance could be rescued. By pretreating EZH2-deficient CRC cells with BPTES, we found that BPTES treatment significantly reversed the decreased cell death phenotype caused by EZH2 deletion or inhibition (Fig. 6A-D). Furthermore, BPTES and EZH2 silencing/inhibition synergistically inhibited the proliferation rate of $\mathrm{CRC}$ cells (Fig. 6E, F). Colony formation assays further confirmed the synergistic effect of the
EZH2 inhibitor and GLS inhibitor (Fig. 5G, H and Supplementary Fig. 6E). Then, we wondered whether other molecules that modulate GLS activity also exhibit a synergistic effect with EZH2 inhibitors; one such molecule is mTOR (Supplementary Fig. $6 \mathrm{~A}$ ), which promotes GLS activity and glutamine metabolism [28, 29]. The results showed that the combined treatment of rapamycin and GSK126 significantly inhibited cancer cell proliferation and colony formation (Supplementary Fig. 6B-D). Collectively, these data suggest that targeting glutamine metabolism has the potential to enhance the efficacy of EZH2 inhibitors and reduce CRC tolerance.

The EZH2-GLS axis regulates glucose-deprivation-induced cell death by modulating intracellular GSH levels

As a key component of redox balance, ROS plays an important role in the cell death induced by glucose deprivation $[1,30]$. In this study, we first used the ROS scavenger NAC to treat glucosedeprived cells and found that glucose-deprivation-induced cell death was significantly attenuated by NAC (Fig. 7A); these results indicated that glucose-deprivation-induced cell death in CRC was mediated by ROS. Furthermore, EZH2 knockdown or inhibition significantly reduced intracellular ROS accumulation under glucose deprivation conditions (Fig. 7B, C), which was consistent with the cell death phenotype (Fig. 2). During glutamine metabolism, GLS converts intracellular glutamine into glutamate, which is used to exchange extracellular cystine through $\mathrm{xCT}$, thereby promoting GSH synthesis and preventing excessive ROS accumulation $[30,31]$. Our results showed that EZH2 knockdown or inhibition significantly increased the intracellular GSH content and that BPTES treatment attenuated the increase in GSH content and the decrease in ROS levels caused by EZH2 deletion under glucose deprivation conditions (Fig. 7D-G). Collectively, these data suggest that the EZH2-GLS axis regulates glucose vulnerability by modulating the intracellular GSH and ROS levels.

\section{DISCUSSION}

Metabolic reprogramming is a hallmark of malignantly transformed cells, making them highly dependent on some nutrients such as glucose [32]. However, during cancer progression or clinical drug intervention, local nutrient deficiency inevitably occurs in cancer tissues. In these cases, how cancer cells adapt to nutrient deprivation to survive and whether epigenetic plasticity is involved in this process are meaningful questions worth exploring in depth. Here through conditional screening of an epigenetic sgRNA library, we found that EZH2 played an important role in the tolerance of colorectal cancer cells to glucose deprivation. In cancer cells facing glucose limitation, the expression of EZH2 was downregulated and $\mathrm{EZH} 2$ deficiency attenuated glucosedeprivation-induced cell death and cell cycle arrest. In solid tumors, EZH2 generally exists as an oncogene, mainly through promoting proliferation, anti-apoptosis, and other biological processes [11]. Although our previous studies demonstrated the cancer-promoting role of EZH2 in the occurrence and progression of colorectal cancer in mice models, this study about glucose deprivation suggested that loss-of-EZH2 can also play a cancer 
A

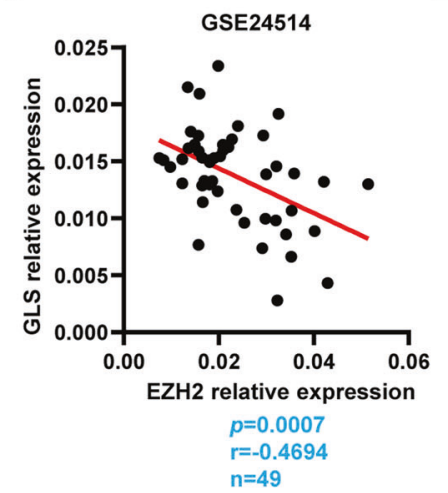

D

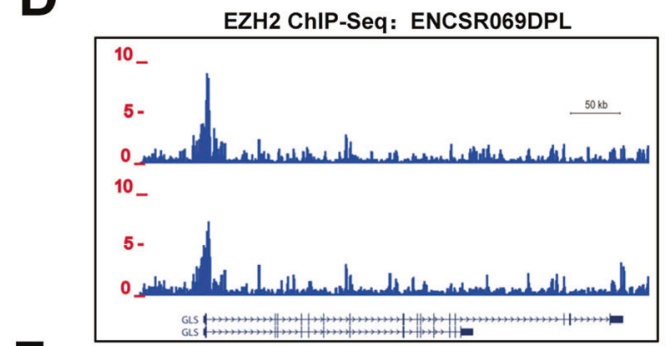

$\mathbf{F}$

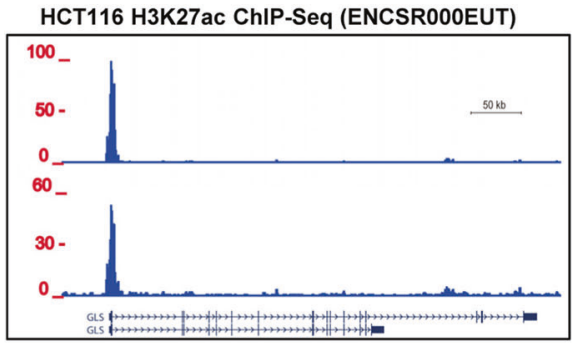

GSE106582

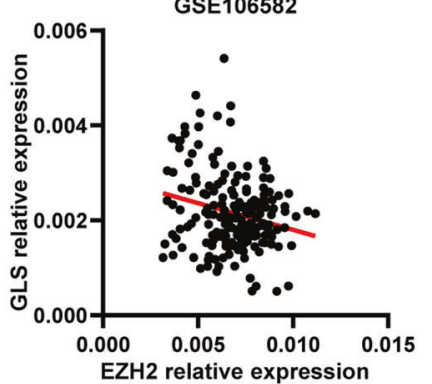

$p=0.0012$

$r=-0.2310$

$n=194$
C

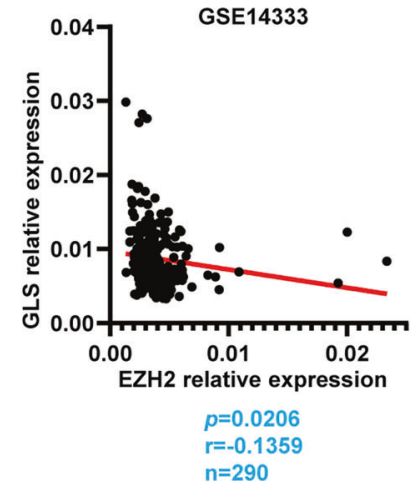

E

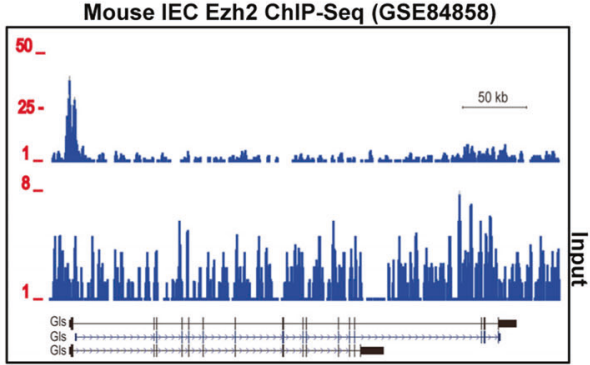

G

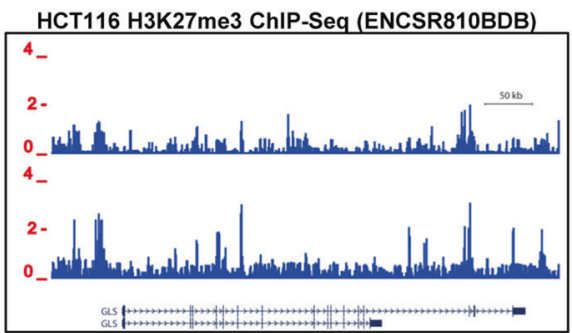

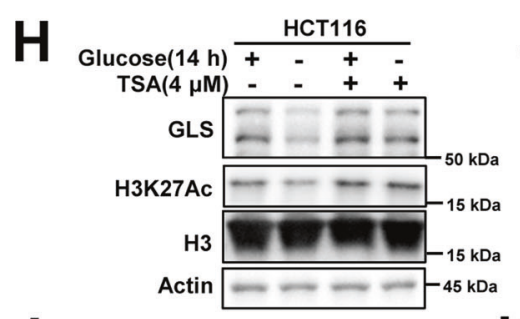

I

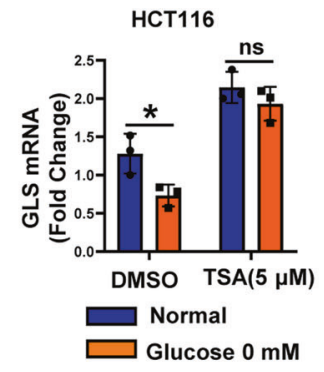

$\mathbf{J}$

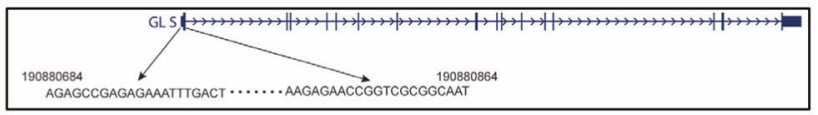

K

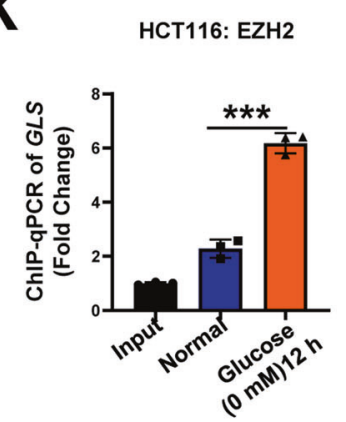

$\mathbf{L}$

HCT116: H3K27AC

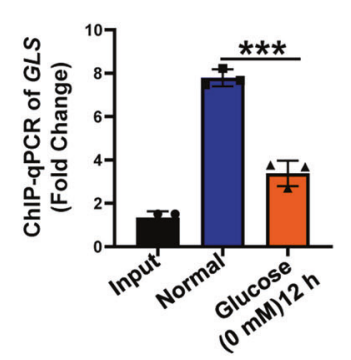

promoting role in certain conditions (Supplementary Fig. 7). The contradiction of these two different roles enlightened us that the occurrence and progression of cancer are different, and heterogeneity of cancer tissues should be taken into consideration under specific conditions. Further, we showed that EZH2 was indeed highly expressed in cancer tissues, but the prognosis of patients was not affected by the expression of EZH2, which was consistent with the conclusion of the previous study [33].

Targeting EZH2 to treat cancer is a hot spot in current clinical research and some inhibitors have been approved by FDA to treat 
Fig. 4 GLS is a direct target gene of EZH2 in colorectal cancer cells. A-C Scatter plots showing the correlation between EZH2 and GLS mRNA levels in human colorectal cancer (GSE24514, GSE14333, and GSE106582). Statistical analysis was performed using a two-tailed Student's $t$-test. $r$, Pearson's correlation coefficient. D Schematic representation of the binding site of human EZH2 in GLS promoter as mapped in ENCODE database (ENCSR069DPL). E Schematic representation of the binding site of mouse EZH2 in Gls promoter of primary colon epithelial cells isolated from wild-type mice (GSE84858). F, G Schematic representation of the binding of H3K27ac (F) and H3K27me3 (G) on the GLS gene locus in HCT116 cells with the ENCODE project. H, I WB and RT-qPCR of GLS levels in CRCs pretreated with DMSO or HDAC inhibitor (TSA, $4 \mu \mathrm{M})$, followed by glucose deprivation treatment $(0 \mathrm{mM}, 16 \mathrm{~h})$. J Location of ChIP-qPCR primers on GLS promoter. K, L ChIP-qPCR analysis of EZH2 and H3K27Ac on GLS promoter in HCT116 cells with or without glucose deprivation treatment $(0 \mathrm{mM}, 12 \mathrm{~h})$. In I, K, and L, data are mean \pm s.d., $n=3$ independent experiments; $P$-values were calculated using two-tailed unpaired Student's $t$-test. ${ }^{*} P<0.05,{ }^{* *} P<0.01,{ }^{* * *} P<$ 0.001 ; ns, non-significant.
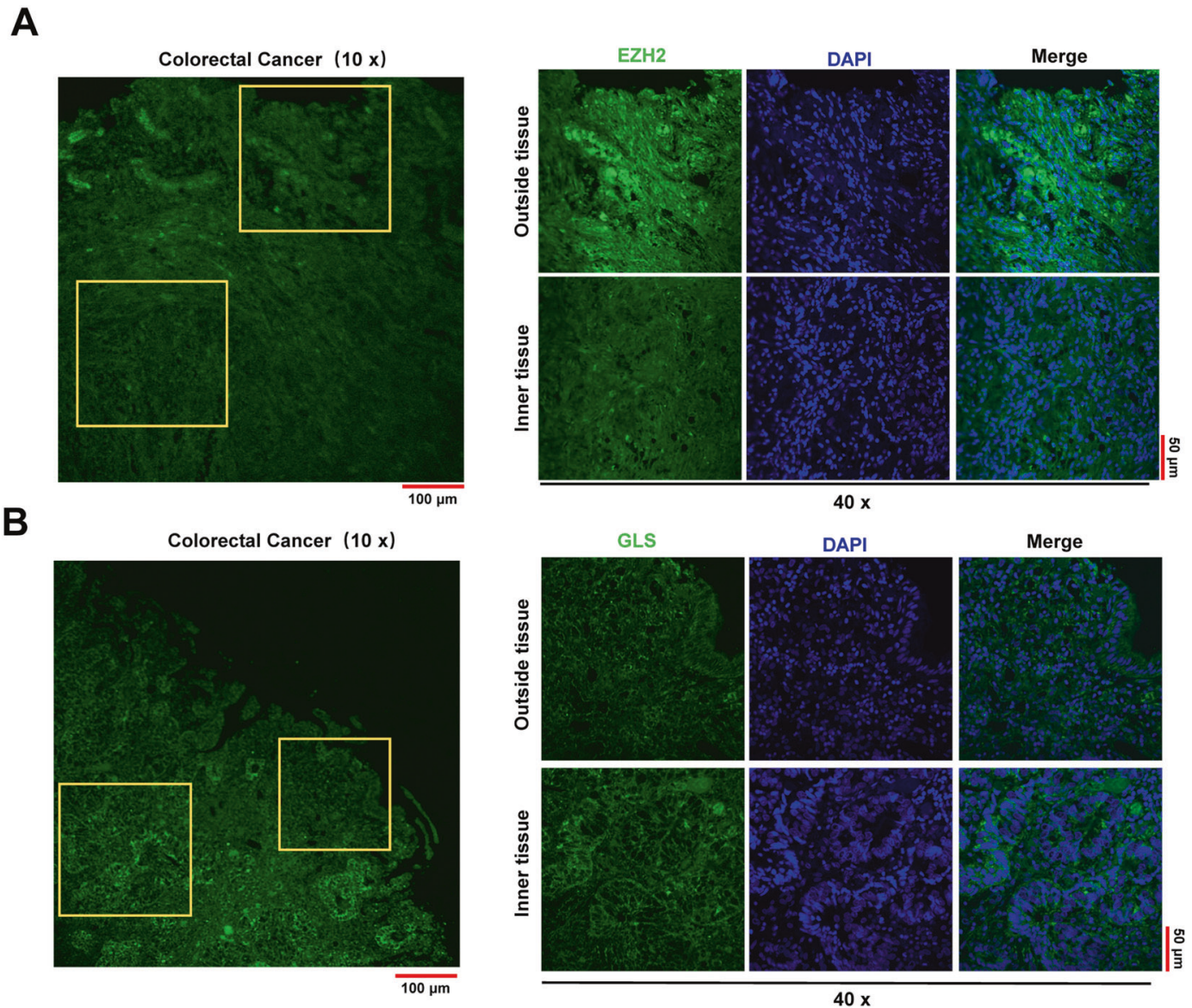

Fig. 5 GLS expression has a negative spatial correlation with EZH2 in colorectal cancer tissues. A, B Immunofluorescence of EZH2 (A) and GLS (B) in human colorectal cancer samples. DAPI is used to indicate the nucleus.

cancer [34]. However, the resistance of cancer cells to EZH2 inhibitors and the underlying mechanisms still need to be explored. In this study, we showed the tumor-suppressive function of EZH2 was mediated by inhibiting the expression of GLS, which played a vital role in cancer metabolic reprogramming. These results might be an explanation for the resistance of cancer cells to EZH2 inhibitors. Mechanistically, we found the metabolismrelated gene GLS could be directly targeted by EZH2. Although EZH2 expression was downregulated under glucose deprivation, the binding of EZH2 on the promoter region of GLS was significantly enhanced, leading to the downregulation of GLS expression. Meanwhile, the loss of EZH2 function resulted in the upregulation of GLS expression, and GLS expression was no longer reduced by glucose deprivation. As a key enzyme of glutamine metabolism, GLS functions as a cancer driver and can also be a therapeutic target in cancer. Under glucose deprivation ROS was accumulated in cancer cells due to the rapidly declining GSH level and shutting down the pentose phosphate pathway. Then the importance of increased GLS induced by EZH2 deficiency was amplified, as GLS converts glutamine to glutamate, which can be used to exchange cystine from extracellular regions and facilitate the synthesis of glutathione to clear ROS. Furthermore, we also proved that the inhibitors that target GLS activity or upstream regulators of glutamine metabolism like mTOR showed synergistic effects with EZH2 inhibitors.

This work and previous studies suggested that the heterogeneity and the plasticity of cancer cells make them respond differently to the same treatment and the existence of this phenomenon to some extent explains the drug resistance to single-target treatments [35-37]. In the context of precision medicine, a better understanding of the heterogeneity of cancer tissues will facilitate the collaborative medication of cancer [38]. Of course, there are still some unresolved issues in our study. For example, cell apoptosis induced by BPTES is also weakened by the 
A

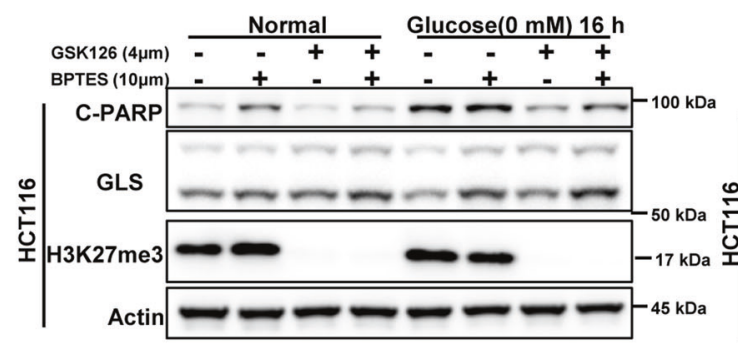

C

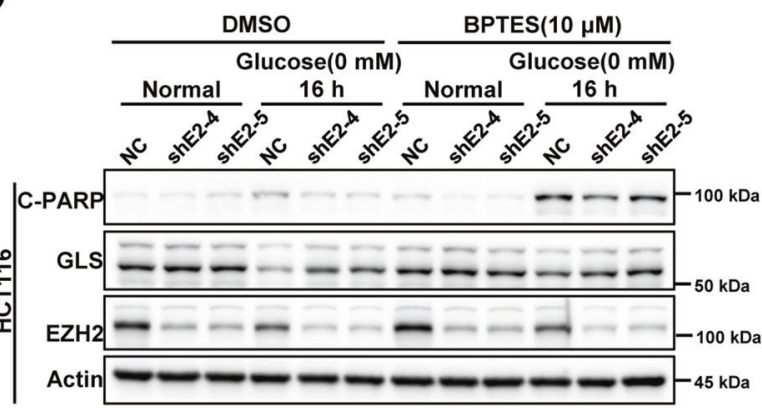

B

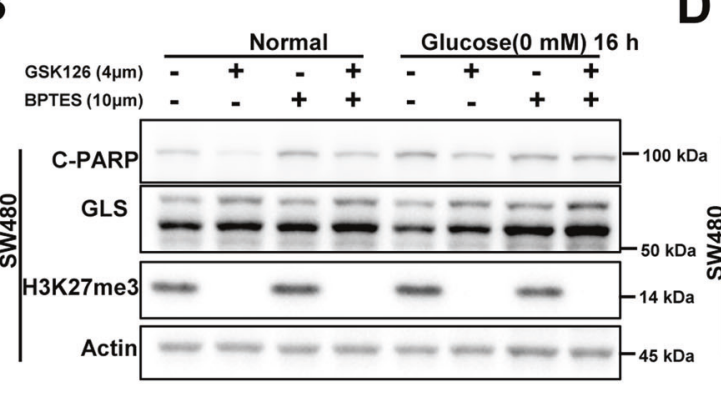

D

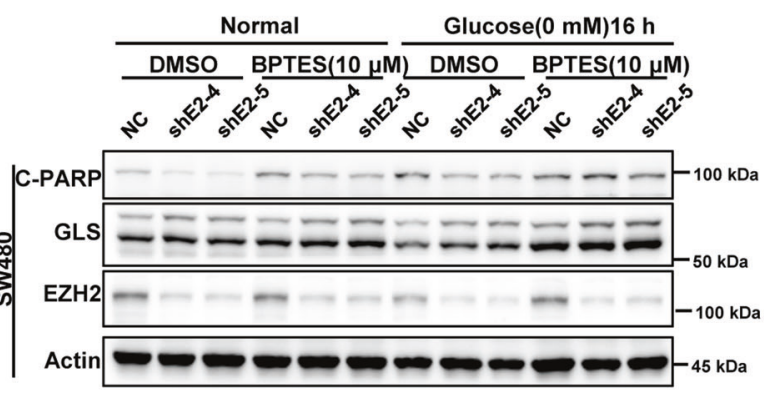

E

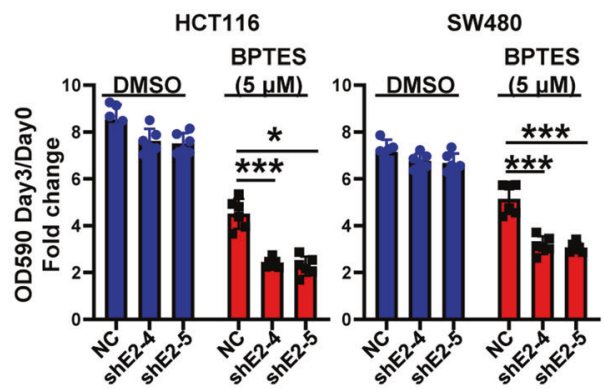

$\mathbf{G}$

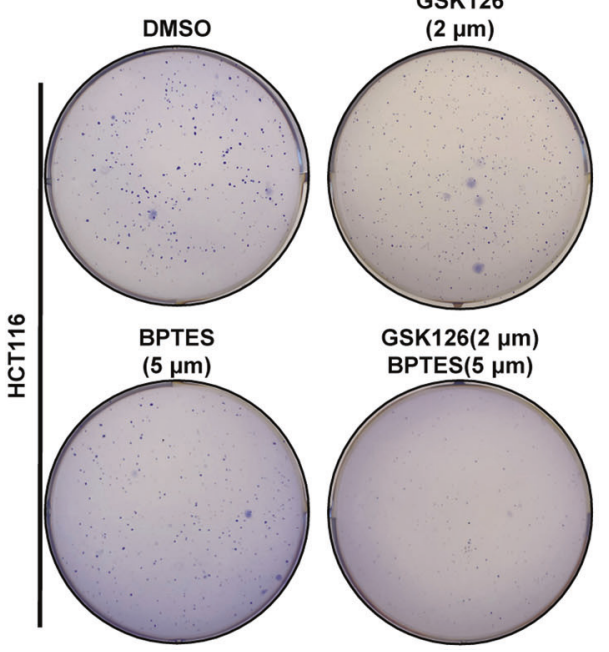

$\mathbf{F}$

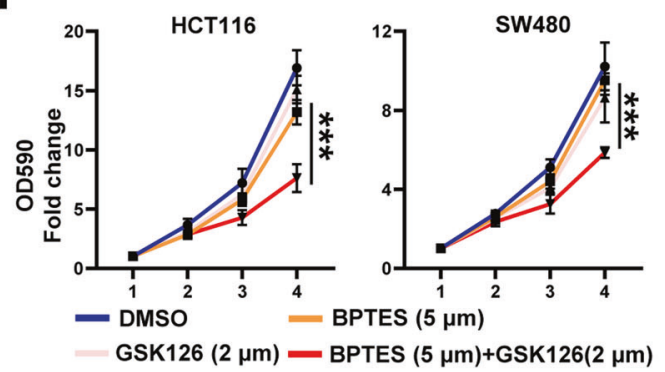

H

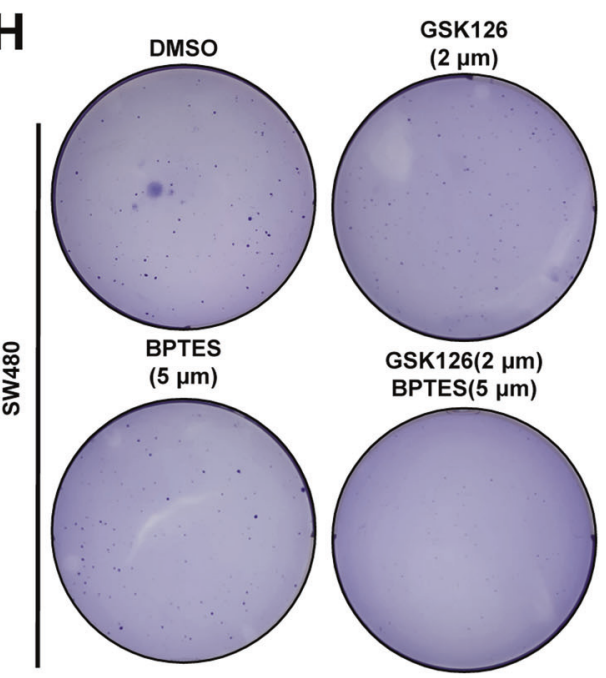

loss of EZH2, which HDAC molecule is responsible for the deacetylation of the H3K27ac in the GLS promoter. Taken together, our study shows the tumor-suppressive function of EZH2 under nutrient-deficient conditions, which partially explains why the clinical expression of EZH2 does not affect the prognosis of CRC patients and reveals a possible mechanism of the resistance of cancer cells to EZH2 inhibitors from the perspective of metabolism. 
Fig. 6 GLS is involved in the resistance of EZH2-deficient cells to glucose deprivation. A, B Immunoblotting for cleaved-PARP and GLS expression in HCT116 (A) or SW480 (B) cells pretreated with or without GSK126 $(4 \mu \mathrm{M}, 48 \mathrm{~h})$ or/and BPTES (10 $\mu \mathrm{M}, 48 \mathrm{~h})$, followed by glucose deprivation treatment $(0 \mathrm{mM}, 16 \mathrm{~h})$. Actin is used as a loading control. C, D Immunoblotting for cleaved-PARP and GLS expression in HCT116 NC and shEZH2 cells (C) or SW480 NC and shEZH2 cells (D) cells pretreated with DMSO or BPTES $(10 \mu \mathrm{M}, 48 \mathrm{~h})$, followed by glucose deprivation treatment $(0 \mathrm{mM}, 16 \mathrm{~h})$. E Three-day growth of HCT116 NC and shEZH2 cells or SW480 NC and shEZH2 cells in presence or not of $5 \mu \mathrm{M}$ BPTES. F Growth curves of HCT116 or SW480 cells in presence or not of $2 \mu \mathrm{M}$ GSK126 or/and $5 \mu \mathrm{M}$ BPTES. G, H Colony formation of HCT116 (G) or SW480 (H) cells in presence or not of $2 \mu \mathrm{M}$ GSK126 or/and $5 \mu \mathrm{M}$ BPTES. The experiments in A-D and G, $\mathbf{H}$ were repeated twice independently with similar results. In $\mathbf{E}, \mathbf{F}$, data are mean \pm s.d., $n=3$ independent experiments; $P$-values were calculated using two-tailed unpaired Student's $t$-test or two-way ANOVA test. ${ }^{*} P<0.05,{ }^{* *} P<0.01,{ }^{* * *} P<0.001$.

\section{MATERIALS AND METHODS \\ Cell culture and reagents}

All cancer cell lines were obtained from the American Type Culture Collection (ATCC, Manassas, VA, USA). All cell lines were free of mycoplasma contamination (tested by the PCR). Cells were cultured in Dulbecco's modified Eagle medium (DMEM) (Thermo Fisher Scientific, Waltham, MA, USA) with $10 \%$ fetal bovine serum (Biological Industries, Kibbutz Beit Haemek, Israel) at $37^{\circ} \mathrm{C}$ with $5 \% \mathrm{CO}_{2}$. For glucose deprivation experiments, cells were cultured in $0 \mathrm{mM}$ Glucose DMEM with 10\% FBS for indicated time and cells were washed by PBS before medium changing. DMEM without glucose was obtained from Thermo Fisher Scientific (11966-025). GSK126 (HY-13470), BPTES (HY-12683), TSA (HY-15144) were got from MedChemExpress (MCE) (NJ, USA). DCFH-DA Reactive Oxygen Species Assay Kit, GSH, and GSSG Assay Kit were purchased from Beyotime Institute of Biotechnology (Shanghai, China). NAC (A7250) was purchased from Sigma-Aldrich (St. Louis, MO, USA).

\section{Stable cell lines generation}

EZH2 short hairpin RNA (shRNA) were constructed with pLKO.1 system and lentiviral plasmid over expressing EZH2 was purchased firm Hanbio (Shanghai, China). Cell lines with specific gene silence or overexpression were generated as previously described. In brief, HEK293T cells were transfected either with pLKO.1 or pLV plasmid, together with $\Delta 8.9$ and VSVg third-generation lentiviral packaging system using Lipofectamine 2000 reagent (Thermo Fisher Scientific) according to the manufacturer's instructions. After transfection, lentivirus particles in the medium were collected every $24 \mathrm{~h}$, and after three collections the medium was filtered, then the target cell lines were infected. At $48 \mathrm{~h}$ after infection, $2 \mu \mathrm{g} / \mathrm{ml}$ puromycin was added to obtain stable cell lines with successful transduction. The sequences of shRNAs used in this study are listed in Supplementary Table 4.

\section{Western blotting}

Western blotting was conducted as previously described [15, 39]. The primary antibodies and concentrations used for Western blotting were following: EZH2 (1:1000, 5246 S, Cell Signaling Technology, Danvers, MA, USA), H3K27me3 (1:1,000, $9733 \mathrm{~S}$, Cell Signaling Technology), C-PARP (1:2,000, 5625 S, Cell Signaling Technology), GLS (1:1,000, $56750 \mathrm{~T}$, Cell Signaling Technology), Actin (1:10,000, AP0060, Bio-world, Nanjing, China).

\section{Reactive oxygen species (ROS) and GSH assay}

For ROS measurement, colorectal cancer cells were seeded in a 12-well plate and treated with corresponding conditions. After the treatment, the medium in the well was transferred to the corresponding EP tube, and then $400 \mathrm{ul}$ medium was added back to the original well. DCFH-DA was added to the wells at a ratio of $1: 1,000$, mixed and incubated at $37^{\circ} \mathrm{C}$ for $15 \mathrm{~min}$. Then the dye solution was removed, the medium in the corresponding EP tubes was added back to the corresponding wells, and observation and photographing were performed through a fluorescence microscope (Olympus IX70). To detect GSH content, cells were treated as indicated and prepared for measurement of glutathione using the GSH and GSSG Assay kit according to the manufacturer's instructions. The concentration of GSH was calculated using a standard curve.

\section{RNA-Seq and ChIP-qPCR analysis}

Total RNA was subjected to MGI2000 performed by BGI Tech Solutions Co., Ltd. Transcriptomic reads were mapped to reference genome (mm10) using Bowtie software, and gene expression levels were quantified using the RSEM software package. The list of significantly affected genes was obtained by setting a false discovery rate (FDR) threshold of $0.001, P$-value
$<0.0,1$ and fold changes greater than 1.2 or $\log 2 \mathrm{Fc}<-0.75$. Manual curation of GOs was performed using KEGG or Enrichr and visualized in the form of a heatmap using - Log10( $P$-value). Gene expression datasets were deposited into the GEO database (GSE159351 and GSE159352). The ChIP assays were performed using the Magnetic ChIP kit (Millipore). The procedure was as described in the kit provided by the manufacturer. Briefly, isolated HCT116 cells were fixed by $1 \%$ formaldehyde, fragmented by sonication. EZH2 (Cell Signaling Technology, 5246 S) and H3K27AC (Cell Signaling Technology, 8173S) were then used for immunoprecipitation. After washing and reverse-crosslinking, the precipitated DNA was amplified by primers and quantified by the GPCR. Primer sequences can be found in Supplementary Table 4.

\section{Cell number assays and colony formation assay}

The cells at $100 \%$ confluency were seeded in 12-well plate 1 day before treatment to measure living cells. After treatment as indicated, cells were trypsinized and collected in a new EP tube and stained with $0.4 \%$ Trypan Blue Solution. The number and proportion of dead and living cells were calculated using Countessll FL. To measure cell viability, cells were seeded in a 96-well plate 1 day before treatment. Following the treatment with the appropriate drugs as indicated, cells were exposed to $10 \mu \mathrm{l}$ resazurin sodium salt. After incubation for $2 \mathrm{~h}$, the plate was analyzed using the Paradigm Detection Platform (Beckman, CA, USA). Colony formation assay was done as previously described [39].

\section{Specimens}

Paraffin-embedded colorectal cancer tissues were collected from patients with CRC who had undergone routine surgery at the Nanfang Hospital, Southern Medical University (Guangzhou, China) and volunteered to provide samples for research.

\section{Immunofluorescence}

Immunofluorescence analysis of EZH2 and GLS was performed on paraffinembedded tissue from colorectal cancer patients. To expose target proteins, antigen retrieval was performed using $10 \mathrm{mM}$ sodium citrate (pH 6.0), microwaved for 8-15 min. Following antigen retrieval, tissues were blocked in $3 \% \mathrm{H}_{2} \mathrm{O}_{2}$-methanol for $15 \mathrm{~min}$ at room temperature, washed with $\mathrm{ddH}_{2} \mathrm{O}$ and PBS, and then probed with EZH2 antibody $(5246 \mathrm{~S})$ or GLS antibody (56750 T) diluted in 3\% BSA-PBS at a dilution of 1:40 for $12 \mathrm{~h}$ at $4^{\circ} \mathrm{C}$ in a humidified chamber. Tissues were washed extensively in PBST and incubated with an FITC-conjugated secondary antibody (Thermo Fisher Scientific) for $2 \mathrm{~h}$. The nuclei were labeled with DAPI (Thermo Fisher Scientific), and fluorescence was monitored using a confocal microscope (Olympus).

\section{Survival analysis and gene expression correlation analysis}

The READ and COAD datasets of the TCGA database were used for expression and survival analysis [40]. Expression profiles of GSE24514, GSE14333, and GSE106582 were downloaded from GEO [41-43]. Statistical computations were performed using GraphPad Prism8. Statistical significance was assessed by One-way ANOVA; the data were considered not significant for $p>0.05$. Correlation values between two genes were determined by iterating through each probe associated with gene EZH2 and comparing it to probe associated with gene GLS. Statistical significance was determined by the Pearson correlation coefficients test.

\section{Mice experiments}

All mice were maintained in a specific-pathogen-free (SPF) facility, and experimental procedures were approved by the institutional biomedical research ethics committee of the Shanghai Institutes for Biological 

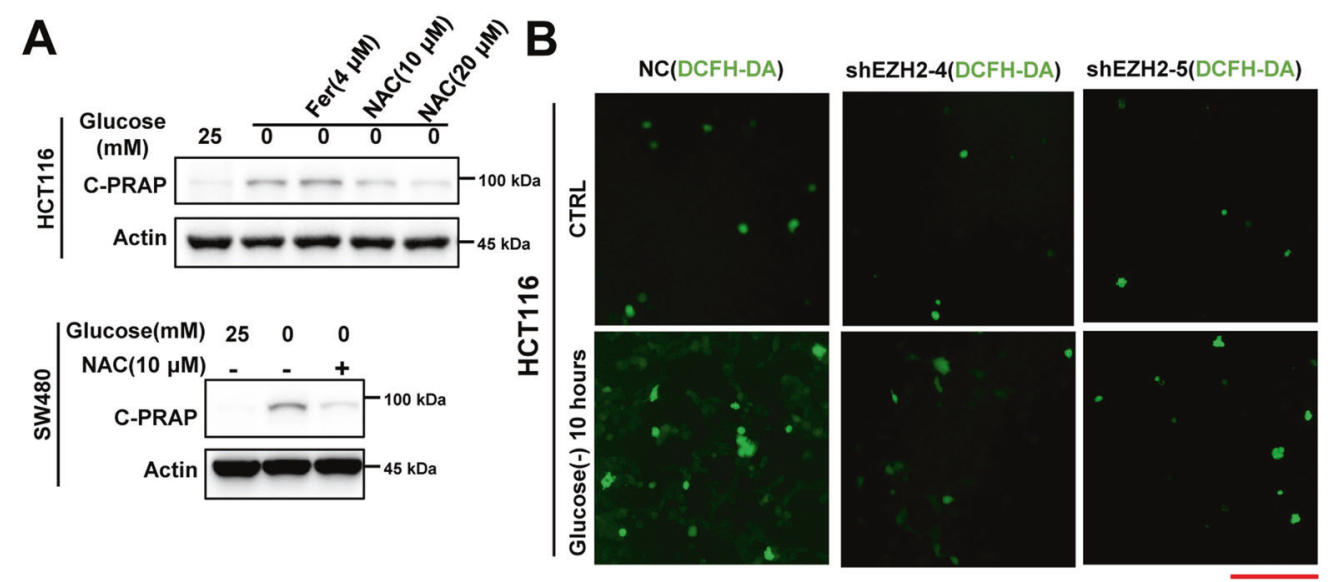
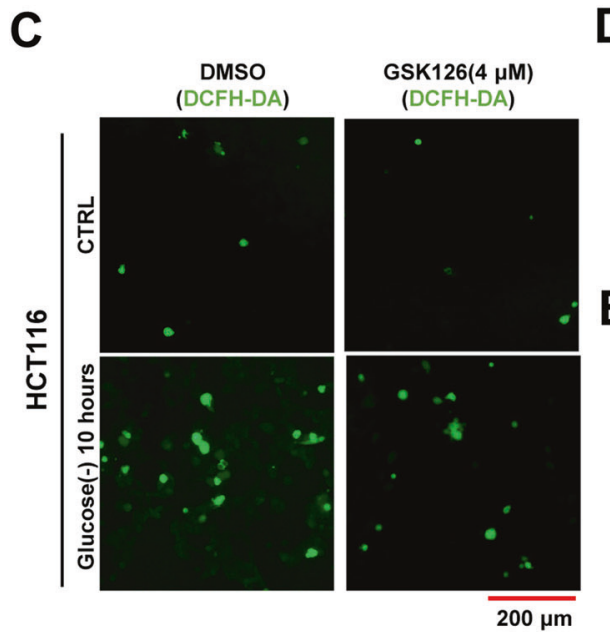

$\mathbf{F}$

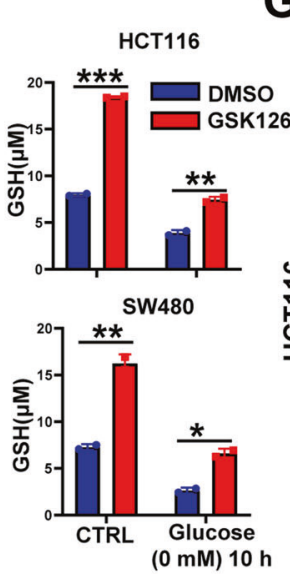

$\mathbf{G}$
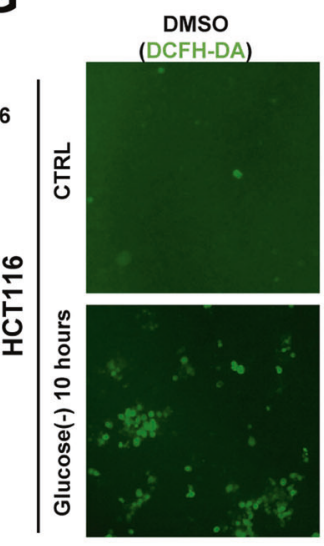

D

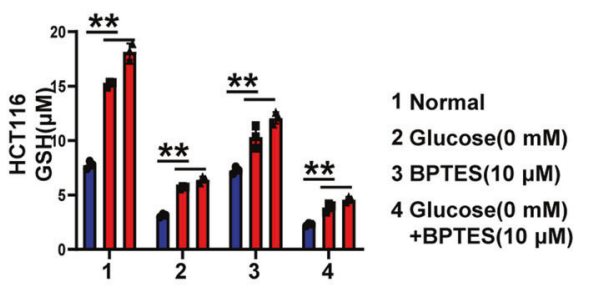

$200 \mu \mathrm{m}$

E
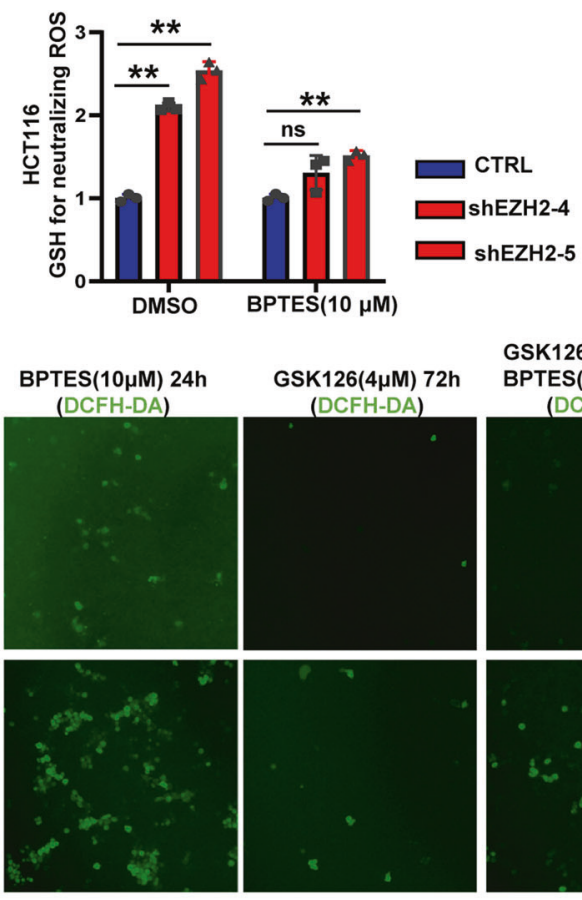

GSK126(4 $\mu \mathrm{M}) 72 \mathrm{~h}$ BPTES $(10 \mu \mathrm{M}) 24 \mathrm{~h}$

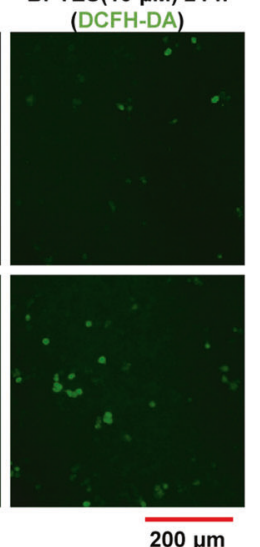

Fig. 7 The EZH2-GLS axis regulates glucose-deprivation-induced cell death by modulating intracellular GSH levels. A Immunoblotting for cleaved-PARP in HCT116 or SW480 cells cultured with or without glucose deprivation $(0 \mathrm{mM}, 16 \mathrm{~h})$ in presence or not of $4 \mu \mathrm{M}$ Fer or $10 \mu \mathrm{M} /$ $20 \mu \mathrm{M}$ NAC ( $n=2$ independent experiments). B DCFH staining of HCT116 NC and shEZH2 cells under normal condition or glucose deprivation $(0 \mathrm{mM}, 10 \mathrm{~h})$. C DCFH staining of HCT116 cells pretreated with DMSO or GSK126 $(4 \mu \mathrm{M}, 48 \mathrm{~h})$, followed by glucose deprivation treatment $(0 \mathrm{mM}, 10 \mathrm{~h})$. D Intracellular GSH content of HCT116 cells pretreated with DMSO or BPTES $(10 \mu \mathrm{M}, 48 \mathrm{~h})$, followed by glucose deprivation treatment $(0 \mathrm{mM}, 10 \mathrm{~h})$. E Intracellular GSH content for neutralizing ROS in HCT116 NC and shEZH2 cells pretreated with DMSO or BPTES $(10 \mu \mathrm{M}, 48 \mathrm{~h})$, followed by glucose deprivation treatment $(0 \mathrm{mM}, 10 \mathrm{~h})$. F Intracellular GSH content of HCT116 or SW480 cells pretreated with DMSO or GSK126 $(4 \mu \mathrm{M}, 48 \mathrm{~h})$, followed by glucose deprivation treatment $(0 \mathrm{mM}, 10 \mathrm{~h})$. G DCFH staining of HCT116 cells pretreated with or without BPTES $(10 \mu \mathrm{M}, 24 \mathrm{~h})$ or/and GSK126 $(4 \mu \mathrm{M}, 72 \mathrm{~h})$, followed by glucose deprivation treatment $(0 \mathrm{mM}, 10 \mathrm{~h})$. The experiments in A-C and $\mathbf{G}$ were repeated twice independently with similar results. In $\mathbf{D}-\mathbf{F}$, data are mean \pm s.d., $n=3$ independent experiments; $P$-values were calculated using two-tailed unpaired Student's $t$-test. ${ }^{*} P<0.05$, ${ }^{* *} P<0.01,{ }^{* *} P<0.001$; ns, non-significant; scale bars, $200 \mu \mathrm{m}$. 
Sciences or Institute of Zoology, Chinese Academy of Sciences and Animal Ethics Committee of Southern Medical University. EZH2 ${ }^{\mathrm{IEC}-1-}$ mice were generated by crossing EZH2 Flox mice [44] with Villin-Cre [45]. Descendants of $\mathrm{APC}^{\mathrm{min} /+}$ mouse [46] and EZH2 ${ }^{\mathrm{IEC}-1-}$ are used to research spontaneous colon cancer. In AOM/DSS experiments, mice were first treated with a single dose of AOM (Sigma), and then 1\% DSS (molecular weight, 36-50 kDa; MP Biomedicals, 160110) was given in the drinking water for 4 days, followed by 2 weeks of regular drinking water. The DSS treatment was repeated for two additional cycles, and mice were sacrificed 10 weeks after AOM injection. All mice were maintained on a C57BL/6 background.

\section{Statistical analysis}

All experiments were independently performed three times unless otherwise stated. Data are presented as mean \pm standard deviation (s.d.). Pearson correlation coefficients were used to evaluate the relationships between EZH2 and GLS expression. Statistical significance was determined by Student's $t$-test, One-way ANOVA, Log-rank test, or Fisher's exact test. For all statistical tests, the 0.05 level of confidence (two-sided) was accepted for statistical significance.

\section{REFERENCES}

1. Liu X, Olszewski K, Zhang Y, Lim EW, Shi J, Zhang X, et al. Cystine transporter regulation of pentose phosphate pathway dependency and disulfide stress exposes a targetable metabolic vulnerability in cancer. Nat Cell Biol. 2020;22:476-86.

2. Pavlova NN, Thompson CB. The emerging hallmarks of cancer metabolism. Cell Metab. 2016;23:27-47.

3. Kerr EM, Gaude E, Turrell FK, Frezza C, Martins CP. Mutant Kras copy number defines metabolic reprogramming and therapeutic susceptibilities. Nature 2016;531:110-3.

4. Mai WX, Gosa L, Daniels VW, Ta L, Tsang JE, Higgins B, et al. Cytoplasmic p53 couples oncogene-driven glucose metabolism to apoptosis and is a therapeutic target in glioblastoma. Nat Med. 2017;23:1342-51.

5. Thai M, Graham NA, Braas D, Nehil M, Komisopoulou E, Kurdistani SK, et al. Adenovirus E4ORF1-induced MYC activation promotes host cell anabolic glucose metabolism and virus replication. Cell Metab. 2014;19:694-701.

6. Jones PA, Issa JP, Baylin S. Targeting the cancer epigenome for therapy. Nat Rev Genet. 2016;17:630-41.

7. Hyun K, Jeon J, Park K, Kim J. Writing, erasing and reading histone lysine methylations. Exp Mol Med. 2017;49:e324.

8. Dardenne E, Beltran H, Benelli M, Gayvert K, Berger A, Puca L, et al. N-Myc induces an $\mathrm{EZH} 2$-mediated transcriptional program driving neuroendocrine prostate cancer. Cancer Cell. 2016;30:563-77.

9. Mohammad F, Weissmann S, Leblanc B, Pandey DP, Højfeldt JW, Comet I, et al. EZH2 is a potential therapeutic target for H3K27M-mutant pediatric gliomas. Nat Med. 2017;23:483-92.

10. Huang $X$, Yan J, Zhang M, Wang $Y$, Chen $Y$, Fu X, et al. Targeting epigenetic crosstalk as a therapeutic strategy for EZH2-aberrant solid tumors. Cell 2018;175:186-99. e19.

11. Kim KH, Roberts CW. Targeting EZH2 in cancer. Nat Med. 2016;22:128-34.

12. Frankel AE, Liu X, Minna JD. Developing EZH2-targeted therapy for lung cancer. Cancer Discov. 2016;6:949-52.

13. Pusapati RV, Daemen A, Wilson C, Sandoval W, Gao M, Haley B, et al. mTORC1dependent metabolic reprogramming underlies escape from glycolysis addiction in cancer cells. Cancer Cell. 2016;29:548-62.

14. Lee JV, Carrer A, Shah S, Snyder NW, Wei S, Venneti S, et al. Akt-dependent metabolic reprogramming regulates tumor cell histone acetylation. Cell Metab. 2014;20:306-19.

15. Liu Y, Peng J, Sun T, Li N, Zhang L, Ren J, et al. Epithelial EZH2 serves as an epigenetic determinant in experimental colitis by inhibiting TNFalpha-mediated inflammation and apoptosis. Proc Natl Acad Sci USA. 2017;114:E3796-E805.

16. Tan $X$, Zhang Z, Liu P, Yao H, Shen L, Tong JS. Inhibition of EZH2 enhances the therapeutic effect of 5-FU via PUMA upregulation in colorectal cancer. Cell Death Dis. 2020;11:1061.

17. Di W, Weinan $X$, Xin L, Zhiwei $Y$, Xinyue $G$, Jinxue $T$, et al. Long noncoding RNA SNHG14 facilitates colorectal cancer metastasis through targeting EZH2regulated EPHA7. Cell Death Dis. 2019;10:514.

18. Villarreal $P$, Pal R, Vargas $G$. In Vivo epithelial metabolic imaging using a topical fluorescent glucose analog. Methods Mol Biol. 2020;2126:21-31.

19. Lei $A$, Chen $L$, Zhang M, Yang $X, X u L$, Cao N, et al. EZH2 regulates protein stability via recruiting USP7 to mediate neuronal gene expression in cancer cells. Front Genet. 2019;10:422.
20. Moya IM, Halder G. Hippo-YAP/TAZ signalling in organ regeneration and regenerative medicine. Nat Rev Mol Cell Biol. 2019;20:211-26.

21. Wu J, Minikes AM, Gao M, Bian H, Li Y, Stockwell BR, et al. Intercellular interaction dictates cancer cell ferroptosis via NF2-YAP signalling. Nature. 2019;572:402-6.

22. Yoo HC, Park SJ, Nam M, Kang J, Kim K, Yeo JH, et al. A variant of SLC1A5 is a mitochondrial glutamine transporter for metabolic reprogramming in cancer cells. Cell Metab. 2020;31:267-83. e12.

23. Alhopuro P, Sammalkorpi H, Niittymäki I, Biström M, Raitila A, Saharinen J, et al. Candidate driver genes in microsatellite-unstable colorectal cancer. Int J Cancer. 2012;130:1558-66.

24. Jorissen RN, Gibbs P, Christie M, Prakash S, Lipton L, Desai J, et al. Metastasisassociated gene expression changes predict poor outcomes in patients with Dukes Stage B and C colorectal cancer. Clin Cancer Res. 2009;15:7642-51.

25. Consortium EP. The ENCODE (ENCyclopedia Of DNA Elements) project. Science 2004;306:636-40.

26. Lee $P$, Malik D, Perkons N, Huangyang $P$, Khare $S$, Rhoades $S$, et al. Targeting glutamine metabolism slows soft tissue sarcoma growth. Nat Commun. 2020;11:498.

27. Masisi BK, El Ansari R, Alfarsi L, Rakha EA, Green AR, Craze ML. The role of glutaminase in cancer. Histopathology . 2020;76:498-508.

28. Csibi A, Fendt SM, Li C, Poulogiannis G, Choo AY, Chapski DJ, et al. The mTORC1 pathway stimulates glutamine metabolism and cell proliferation by repressing SIRT4. Cell . 2013;153:840-54.

29. Huang T, Liu R, Fu X, Yao D, Yang M, Liu Q, et al. Aging reduces an ERRalphadirected mitochondrial glutaminase expression suppressing glutamine anaplerosis and osteogenic differentiation of mesenchymal stem cells. Stem Cells. 2017:35:411-24.

30. Hu K, Li K, Lv J, Feng J, Chen J, Wu H, et al. Suppression of the SLC7A11/ glutathione axis causes synthetic lethality in KRAS-mutant lung adenocarcinoma. J Clin Invest. 2020;130:1752-66.

31. Huang Y, Dai Z, Barbacioru C, Sadee W. Cystine-glutamate transporter SLC7A11 in cancer chemosensitivity and chemoresistance. Cancer Res. 2005;65:7446-54.

32. Faubert B, Solmonson A, DeBerardinis RJ. Metabolic reprogramming and cancer progression. Science. 2020, https://doi.org/10.1126/science.aaw5473.

33. Vilorio-Marqués L, Martín V, Diez-Tascón C, González-Sevilla MF, Fernández-Villa T, Honrado $\mathrm{E}$, et al. The role of EZH2 in overall survival of colorectal cancer: a metaanalysis. Sci Rep. 2017;7:13806.

34. Julia E, Salles G. EZH2 inhibition by tazemetostat: mechanisms of action, safety and efficacy in relapsed/refractory follicular lymphoma. Future Oncol. 2021;17:2127-40.

35. Turajlic S, Sottoriva A, Graham T, Swanton C. Resolving genetic heterogeneity in cancer. Nat Rev Genet. 2019;20:404-16.

36. Ganesh K. Plasticity in motion: shape-shifting Lgr5(-)cells initiate colorectal cancer metastasis. Cell Stem Cell. 2020;26:469-71.

37. Schmidt F, Efferth T. Tumor Heterogeneity, Single-Cell Sequencing, and Drug Resistance. Pharmaceuticals (Basel). https://doi.org/10.3390/ph9020033.

38. Dienstmann R, Vermeulen L, Guinney J, Kopetz S, Tejpar S, Tabernero J. Consensus molecular subtypes and the evolution of precision medicine in colorectal cancer. Nat Rev Cancer. 2017;17:268.

39. Li N, Xue W, Yuan H, Dong B, Ding Y, Liu Y, et al. AKT-mediated stabilization of histone methyltransferase WHSC1 promotes prostate cancer metastasis. J Clin Invest. 2017;127:1284-302.

40. Tang Z, Li C, Kang B, Gao G, Li C, Zhang Z. GEPIA: a web server for cancer and normal gene expression profiling and interactive analyses. Nucleic Acids Res. 2017;45:W98-W102. W1

41. Tan $X$, Tang $H$, Gong $L$, Xie L, Lei $Y$, Luo $Z$, et al. Integrating genome-Wide association studies and gene expression profiles with chemical-genes interaction networks to identify chemicals associated with colorectal cancer. Front Genet. 2020;11:385.

42. Mahmoud O, Harrison A, Perperoglou A, Gul A, Khan Z, Metodiev MV, et al. A feature selection method for classification within functional genomics experiments based on the proportional overlapping score. BMC Bioinforma. 2014;15:274.

43. Watanabe T, Kobunai T, Yamamoto $Y$, Matsuda K, Ishihara S, Nozawa K, et al. Chromosomal instability (CIN) phenotype, CIN high or CIN low, predicts survival for colorectal cancer. J Clin Oncol. 2012;30:2256-64.

44. Su IH, Basavaraj A, Krutchinsky AN, Hobert O, Ullrich A, Chait BT, et al. Ezh2 controls $B$ cell development through histone $\mathrm{H} 3$ methylation and Igh rearrangement. Nat Immunol. 2003;4:124-31.

45. Madison BB, Dunbar L, Qiao XT, Braunstein K, Braunstein E, Gumucio DL. Cis elements of the villin gene control expression in restricted domains of the vertical (crypt) and horizontal (duodenum, cecum) axes of the intestine. J Biol Chem. 2002;277:33275-83.

46. Yuan H, Li N, Fu D, Ren J, Hui J, Peng J, et al. Histone methyltransferase SETD2 modulates alternative splicing to inhibit intestinal tumorigenesis. J Clin Invest. 2017;127:3375-91. 


\section{ACKNOWLEDGEMENTS}

We thank Li. Lin for technical help and discussion. We thank Feng. Tan, Lisha. Mo, Tao. Xing and Xiaoxin. Ren for critical reading of the manuscript.

\section{AUTHOR CONTRIBUTIONS}

$Y L$ and $C T$ designed, performed most of the experiments and analyzed data. $X G, C W$, $\mathrm{CG}, \mathrm{QL}, \mathrm{YF}$, and $\mathrm{JH}$ helped with the experiments and interpretation of results. $\mathrm{YL}$ conducted bioinformatics analysis. SL, ZW, and AL provided reagents, supported the project, and supervised the study. $\mathrm{YL}$ and $\mathrm{CT}$ wrote the manuscript. All authors have contributed to and approved the final paper.

\section{FUNDING}

This project was supported by "Guangdong gastrointestinal disease research center" (No.2017B020209003), Natural Science Foundation of China (No. 82003291, No. 82002593 and No. 31701174), Outstanding Youth Development Scheme of Nanfang Hospital, Southern Medical University and Natural Science Foundation of Guangdong Province (No. 2020A1515011283).

\section{COMPETING INTERESTS}

The authors declare no competing interests.

\section{ETHICS STATEMENT}

The study was approved by the Ethics Committee of the Nanfang Hospital and Southern Medical University. The data were analyzed anonymously.

\section{ADDITIONAL INFORMATION}

Supplementary information The online version contains supplementary material available at https://doi.org/10.1038/s41419-021-04212-7.

Correspondence and requests for materials should be addressed to Yongfeng Liu, Zhizhang Wang, Aimin Li or Side Liu.

Reprints and permission information is available at http://www.nature.com/ reprints

Publisher's note Springer Nature remains neutral with regard to jurisdictional claims in published maps and institutional affiliations.

(i) Open Access This article is licensed under a Creative Commons Attribution 4.0 International License, which permits use, sharing, adaptation, distribution and reproduction in any medium or format, as long as you give appropriate credit to the original author(s) and the source, provide a link to the Creative Commons license, and indicate if changes were made. The images or other third party material in this article are included in the article's Creative Commons license, unless indicated otherwise in a credit line to the material. If material is not included in the article's Creative Commons license and your intended use is not permitted by statutory regulation or exceeds the permitted use, you will need to obtain permission directly from the copyright holder. To view a copy of this license, visit http://creativecommons. org/licenses/by/4.0/.

(c) The Author(s) 2021 